\title{
Advances in PZT-on-SiN electro-optic modulator platform
}

\author{
John Puthenparampil George ${ }^{1,2,3}$, Koen Alexander ${ }^{1,2}$, Bart Kuyken ${ }^{1,2}$, Dries Van Thourhout ${ }^{1,2}$, \\ Jeroen Beeckman ${ }^{3}$ \\ ${ }^{1}$ Photonics Research Group, INTEC, Ghent University-IMEC, Ghent B-9000, Belgium \\ ${ }^{2}$ Center for Nano-and Biophotonics (NB-Photonics), Ghent University, Ghent B-9000, Belgium \\ ${ }^{3}$ Liquid Crystals and Photonics Group, Ghent University, Ghent University, Ghent B-9000, Belgium \\ e-mail address.John.PuthenparampilGeorge@ugent.be
}

\begin{abstract}
We demonstrate an O-band electro-optic modulator on SiN platform based on ferroelectric PZT thin films. A ring modulator with a small signal bandwidth in excess of $33 \mathrm{GHz}$ is reported. (C) 2018 The Author(s)
\end{abstract}

\section{Introduction}

Electro-optic modulators based on the Pockels effect are highly desirable as they produce pure phase modulation to encode advanced modulation formats [1]. SiN is a passive waveguide platform characterized by its broadband transparency, low loss, and good optical confinement. However, active devices such as modulators, and lasers are hardly available. Due to the centro-symmetric crystal structure of the SiN, -electro-optic interactions based on the Pockels effect are absent. In this work, we demonstrate a high-speed electro-optic Pockels modulator based on SiN platform.

To achieve this, highly electro-optic PZT layers are deposited over the SiN waveguide using a novel chemical solution deposition method [2]. We have reported C-band electro-optic modulation on this platform [3]. Here, we demonstrate measurements performed on the O-band ring resonator (MZI) modulators show a small signal bandwidth beyond $33 \mathrm{GHz}$, and a $\mathrm{V}_{\pi} \mathrm{L}_{\pi}$ of $3.19 \mathrm{~V} \mathrm{~cm}$. The effective electro-optic coefficient is extracted to be 200 $\mathrm{pm} / \mathrm{V}$, which is comparable to state-of-art ferroelectric- Si modulators [4].

\section{Device fabrication}

The waveguides, ring resonators etc. are first patterned into a layer of LPCVD SiN (350 nm) on top of a buried oxide layer $(\approx 3 \mu \mathrm{m})$ on a silicon handle wafer in a CMOS pilot line. Subsequently, $\mathrm{PECVD} \mathrm{SiO}_{2}$ layers $(\approx 1 \mu \mathrm{m})$ are deposited over the waveguide and planarized by chemical mechanical polishing (CMP), to facilitate the PZT deposition. Firstly, a thin intermediate $\mathrm{La}_{2} \mathrm{O}_{2} \mathrm{CO}_{3}$ layer $(\approx 25 \mathrm{~nm})$ is deposited over the planarized waveguide, to serve as seed layer and diffusion barrier for high temperature PZT growth. The PZT layers are deposited in a step of $25 \mathrm{~nm}$ followed by pyrolysis, and annealed in an oxygen ambient, to achieve a total layer thickness of $150 \mathrm{~nm}$. The PZT layers are selectively patterned over the grating couplers by a $\mathrm{SF}_{6}$ based reactive ion etching (RIE) chemistry. The grating couplers are used to interface with chip for optical measurements. Finally, electrical contacts are defined using photolithography, thermal evaporation and lift-off. Figure. 1(a) and Figure 1(b) represent the microscope and SEM cross section image of the fabricated device.
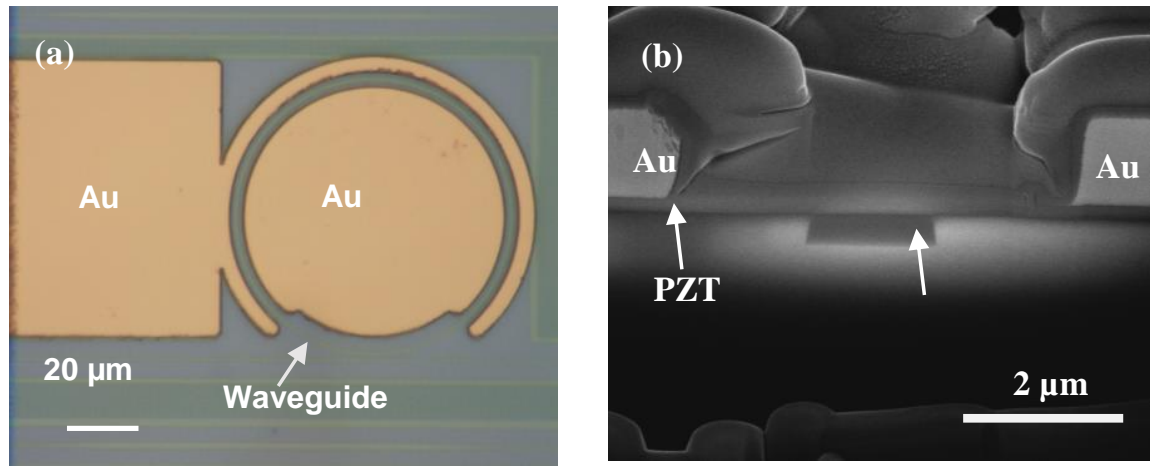

Figure 1: (a) Top view of PZT-on-SiN O-band ring modulator. (b) SEM cross section image of the PZT covered SiN waveguide with pattered gold electrodes. 


\section{Experimental results}

The fabricated O-band ring resonator modulator uses waveguide of width $1600 \mathrm{~nm}$, the electrode separation of 5 $\mu \mathrm{m}$, and the length of the phase shifter section is $195 \mu \mathrm{m}$. The ring has a Q-factor and the free spectral range of 1820 and 3.27, respectively. Since the deposited PZT films show preferential out-of-plane crystal growth without any specific in-plane orientation, therefore it is essential to pole the films to obtain strong electro-optic response. In this case, a DC voltage $(\approx 50 \mathrm{~V})$ above the coercive field is applied to the electrode for 30 minutes. To characterize the static performance of the modulators a set of DC voltages [0 to 6V] are applied to the gold electrodes (Figure 2(a).). The obtained resonance shift as function of applied DC voltage is shown in Figure 2 (b). The modulation efficiency of the current O-band modulator is $3.16 \mathrm{~V} \mathrm{~cm}$, this could be further improved by increasing the PZT thickness. We extracted an effective pockels coefficient of $200 \mathrm{pm} / \mathrm{V}$, which is comparable to the $\mathrm{BaTiO}_{3}$ modulators demonstrated on the Silicon-on-insulator platform [4]. Propagation losses of the PZT-on-SiN waveguides can be as low as 1-2 $\mathrm{dB} / \mathrm{cm}$, when measured with a cut back method.

To demonstrate the high-speed properties of the device, we used a vector network analyzer (VNA) to study its small signal response at high modulation speeds (see Figure $2(\mathrm{~b})$ ). The resulting response shows bandwidth in excess of $33 \mathrm{GHz}$ for the O-band modulators, which is currently limited by the experimental set-up.
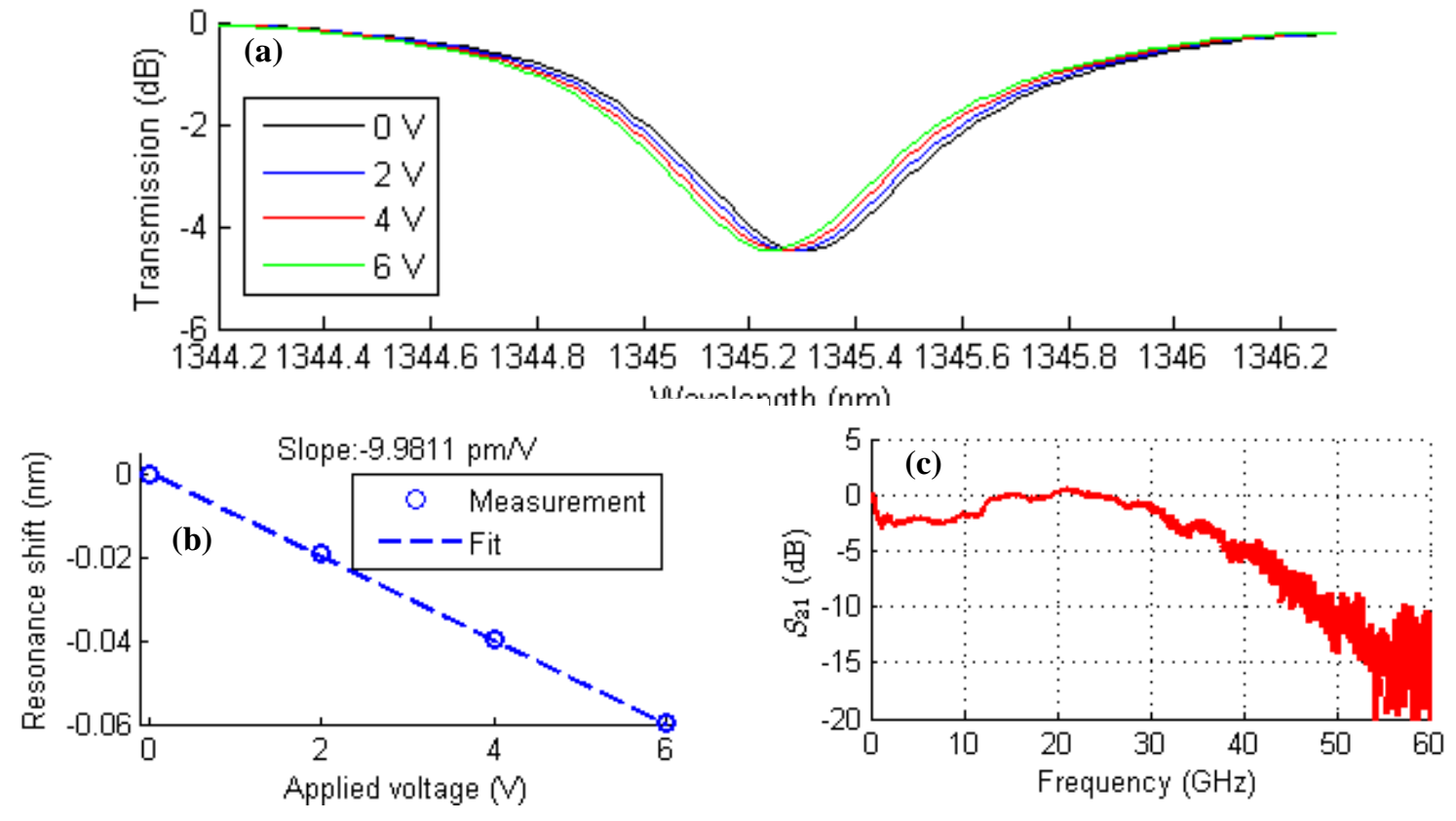

Figure 2: (a) Transmission spectrum of the ring for different DC voltages. (b) Resonance shift as a function of Voltage. (c) Small signal modulation response ring modulator.

\section{Conclusion and future outlook}

In this work, we have demonstrated an O-band high speed ring resonator modulator on the SiN platform, based on integrated electro-optic PZT thin films. The ring modulators demonstrate a small signal bandwidth beyond $33 \mathrm{GHz}$, with a modulation efficiency of $3.16 \mathrm{~V} \mathrm{~cm}$. Further measurements on the devices are ongoing, and it will be presented at the conference.

\section{References}

[1] Seimetz., et al, "High-order modulation for optical fiber transmission," (Springer Verlag, Berlin, Heidelberg, 2009

[2] George, J. P., et al., "Lanthanide-assisted deposition of strongly electro-optic PZT thin films on silicon: Toward integrated active nanophotonic devices," ACS Applied Materials \& Interfaces 7(24), 13350-13359 (2015).

[3] Alexander, K., et al., "Broadband electro-optic modulation using low-loss PZT-on-silicon nitride integrated waveguides," CLEO. JTh5C.7.2017

[4] Xiaong.C et al., "Active silicon integrated nanophotonics: ferroelectric BaTiO3 devices," ACS Applied Materials \& Interfaces 7(24), 13350-13359 (2015) 
